\title{
Giant Circular Dichroism in Individual Carbon Nanotubes Induced by Extrinsic Chirality
}

\author{
A. Yokoyama, M. Yoshida, A. Ishii, and Y. K. Kato* \\ Institute of Engineering Innovation, The University of Tokyo, Tokyo 113-8656, Japan \\ (Received 11 August 2013; revised manuscript received 20 November 2013; published 21 January 2014) \\ Circular dichroism is widely used for characterizing organic and biological materials, but measurements \\ at a single-molecule level are challenging because differences in absorption for opposite helicities are \\ small. Here, we show that extrinsic chirality can induce giant circular dichroism in individual carbon \\ nanotubes, with the degree of polarization reaching $65 \%$. The signal has a large dependence on the \\ incidence angle, consistent with extrinsic-chirality-induced effects in which symmetry is broken by the \\ optical wave vector. We propose that the field-induced charge distribution on the substrate results in an \\ efficient polarization conversion, giving rise to the giant dichroism. Our results highlight the possibility of \\ polarization manipulation at the nanoscale for applications in integrated photonics and novel metamaterial \\ designs.
}

DOI: 10.1103/PhysRevX.4.011005

Subject Areas: Nanophysics, Optics, Photonics

Materials with mirror symmetry breaking exhibit differences in absorption for the two helicities of circular polarization, which is known as circular dichroism (CD). It is a fundamental effect in electromagnetism, and yet it finds broad applications in analytical chemistry, molecular biology, and crystallography for identification and characterization of chiral molecules and solids [1,2]. Despite its common use on macroscopic samples, detection of $\mathrm{CD}$ from single molecules is nontrivial, as the difference in absorption is typically less than a part in a thousand. The use of superchiral optical fields can enhance enantioselectivity $[3,4]$, but CD spectroscopy at a single-molecule level still remains a challenge [5-9]. In contrast to the smallness of the effect in molecules, giant $\mathrm{CD}$ at optical frequencies has been demonstrated in metamaterials [10-13]. Such strong chirality effects are expected to open up novel opportunities in optics and photonics, including negative index media [14], but carefully designed metal nanostructures with appropriate resonances are required.

Here, we report on the observation of giant $\mathrm{CD}$ in individual single-walled carbon nanotubes (CNTs) and show that it arises from extrinsic chirality [12]. Spectrally and spatially resolved CD measurements are performed on single air-suspended CNTs using photoluminescence (PL) for detection, unambiguously revealing circularly polarized absorption originating from the nanotubes. Surprisingly, the degree of polarization reaches a value as high as $65 \%$, an unforeseen level of CD in single nanoparticles. We find that the $\mathrm{CD}$ signal is strongly dependent on the angle of incidence, changing its sign

\footnotetext{
"ykato@sogo.t.u-tokyo.ac.jp
}

Published by the American Physical Society under the terms of the Creative Commons Attribution 3.0 License. Further distribution of this work must maintain attribution to the author(s) and the published article's title, journal citation, and DOI. and vanishing at certain angles. All nanotubes investigated have shown angle-dependent polarization with the same sign, against the expectation that half of the nanotubes show the opposite sign if the nanotube handedness is responsible for the dichroism. Rather, the results are consistent with CD that is induced by extrinsic chirality, where the optical wave vector breaks the mirror symmetry under oblique incidence. We propose a microscopic physical mechanism in which a spatially distributed field-induced charge on the substrate surface causes enhancement or reduction of absorption for circular polarization. The results demonstrate the feasibility of polarization manipulation using extrinsic chirality at the level of a single nanoparticle, opening up the possibility of polarization engineering at the nanoscale.

Our samples are single-walled carbon nanotubes suspended over trenches on silicon substrates. Electron-beam lithography and dry-etching processes are performed to form approximately $5-\mu$ m-deep trenches on (001) $\mathrm{Si}$ wafers. Another electron-beam lithography step defines the catalyst windows, and nanotubes are grown by chemical vapor deposition using ethanol as a carbon source $[15,16]$. The nanotubes are then characterized with a laserscanning confocal microspectroscopy system [17-19]. A wavelength-tunable continuous-wave Ti:sapphire laser is used for excitation, and an objective lens with a numerical aperture of 0.7 and a working distance of $10 \mathrm{~mm}$ focuses the laser and collects the PL at a resolution of approximately $1.5 \mu \mathrm{m}$. An InGaAs photodiode array attached to a spectrometer is used for obtaining PL spectra. A steering mirror scans the laser spot to image and identify wellisolated CNTs, while chiral indices are assigned by PL excitation spectroscopy [20,21]. PL intensity dependence on the linear polarization angle allows the determination of the nanotube axis.

After we identify and characterize individual CNTs, CD measurements are performed in the side excitation 
geometry, as shown in Fig. 1(a). The excitation laser beam is directed at a glancing angle of around $10^{\circ}$ from the sample plane, lightly focused by a lens with a focal length of $75 \mathrm{~mm}$ to an elliptical spot with full widths at half maximum of about $60 \mu \mathrm{m}$ and $20 \mu \mathrm{m}$ along the $x$ and $y$ directions, respectively. The laser is initially linearly polarized along the $y$ axis, and an achromatic quarter-wave plate is placed just before the lens to obtain circular polarization. The sample is mounted on an automated rotation and three-dimensional translation stage to allow

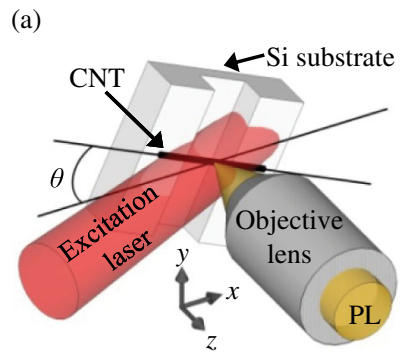

(b)
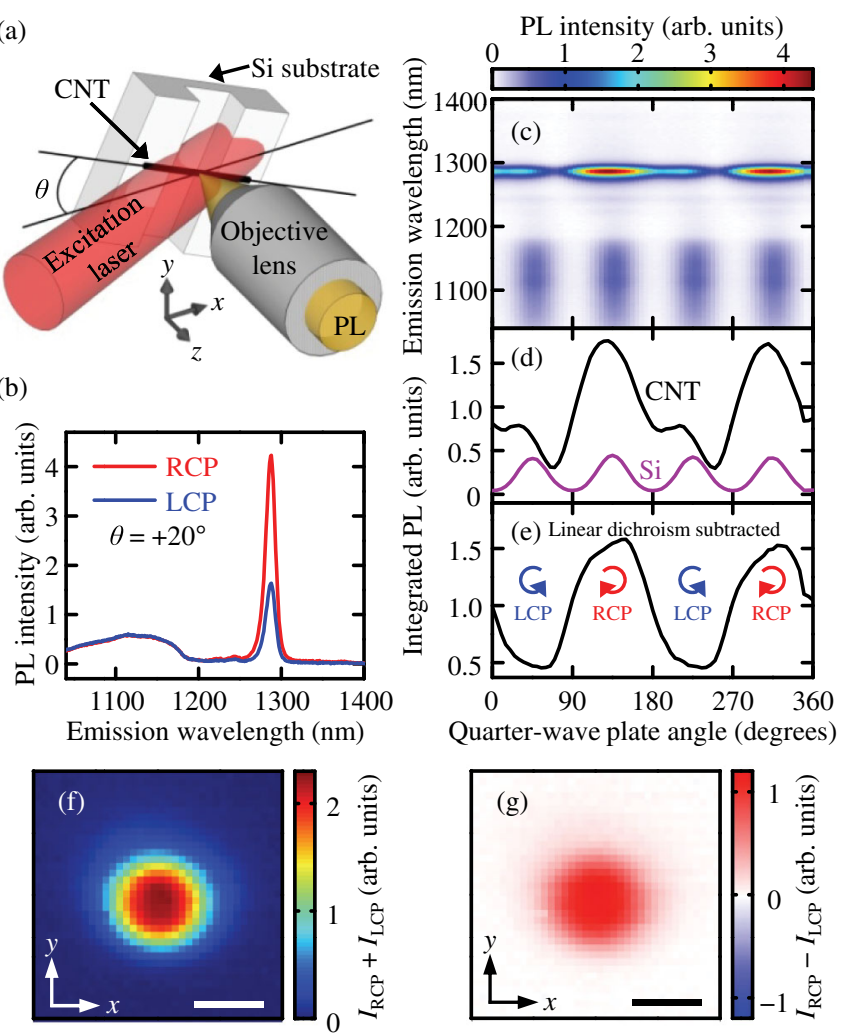

FIG. 1 (color online). (a) A schematic of the experimental geometry. The substrate is parallel to the $x y$ plane, and the optical collection path through the objective lens is along the $z$ axis. (b) PL spectra of an individual nanotube for excitation with RCP (red, upper curve) and LCP (blue, lower curve). (c) PL spectra as a function of the quarter-wave plate angle. The laser is linearly polarized along the $y$ axis before the wave plate. (d) Integrated PL intensity from CNT (black) and Si (purple) as a function of the quarter-wave plate angle. The spectral integration window for the CNT is centered at $1290 \mathrm{~nm}$ and has a width of $40 \mathrm{~nm}$, while $\mathrm{Si}$ PL has been integrated from $1040 \mathrm{~nm}$ to $1200 \mathrm{~nm}$. (e) Linear dichroism for CNT PL is extracted by fitting the data in (d) by two sinusoidal functions with periods of $90^{\circ}$ and $180^{\circ}$, and the component with $90^{\circ}$ periodicity has been subtracted. (f) and (g) Sum and difference images, respectively, of integrated CNT PL for excitation with RCP and LCP. The excitation laser spot is fixed, and the collection spot is scanned to obtain the images. Scale bars are $2 \mu \mathrm{m}$. For (b-g), $\lambda=780 \mathrm{~nm}$ and $P=26 \mathrm{~mW}$ are used for the excitation. The nanotube is at $\theta=+20^{\circ}$ and has a suspended length of $1.3 \mu \mathrm{m}$. in-plane incidence-angle dependence measurements. All measurements are done at room temperature in air.

Figure 1(b) shows typical PL spectra taken at an in-plane angle $\theta=+20^{\circ}$ for excitation with right-circular polarization (RCP) and left-circular polarization (LCP). The excitation wavelength $\lambda$ is tuned to the $E_{22}$ resonance of this $(9,7)$ tube, and a power $P=26 \mathrm{~mW}$ is used. The sharp peak near $1300 \mathrm{~nm}$ is the emission from the CNT, while Si $\mathrm{PL}$ is the weaker luminescence centered around $1100 \mathrm{~nm}$. It is clear that the nanotube PL is modulated by a factor of approximately 2.5 for RCP and LCP.

Such a change in PL intensity can arise from beam deviation with wave plate rotation, but we use an actuatordriven mirror to correct the deviation of the laser spot to below $2 \mu \mathrm{m}$. The fact that Si PL intensity is nearly the same for the two helicities shows that the excitation-intensity changes are insignificant, and therefore the change in CNT PL must arise from laser polarization.

As CNTs have strong linearly polarized absorption $[17,22,23]$, inaccuracies in the angle of the quarter-wave plate can give rise to an artifact. To distinguish linear and circular dichroism signals, we have performed the measurements as a function of the quarter-wave plate angle [Fig. 1(c)]. Linear dichroism gives rise to intensity modulation with a $90^{\circ}$ period, as in the case for Si PL where $p$ polarization has a larger transmission coefficient and thus results in stronger PL [Fig. 1(d)]. In comparison, the nanotube PL intensity modulation has a component with a period of $180^{\circ}$ [Fig. 1(e)], a signature of circularly polarized absorption.

As another possible source of an artifact, we have examined the polarization of the reflected laser. If the polarization is significantly different for RCP and LCP after reflection, and if the reflected laser is partly responsible for the excitation of CNTs, differences in PL intensity for incoming RCP and LCP could occur. We dismiss such an interpretation because we have observed that the reflected polarization is largely $s$ polarized for both helicities, as expected for a glancing reflection from a substrate with a large index of refraction.

Next, we show that the signal is localized at the nanotube position by using PL images under circularly polarized excitation. We define $I_{\mathrm{RCP}}$ and $I_{\mathrm{LCP}}$ to be integrated PL intensity for excitation with RCP and LCP, respectively. We compute the sum $I_{\mathrm{RCP}}+I_{\mathrm{LCP}}$ and the difference $I_{\mathrm{RCP}}-$ $I_{\mathrm{LCP}}$ to construct the images shown in Figs. 1(f) and 1(g), respectively. These images show that the position and the extent of the difference signal overlaps with that of the sum signal. As the observed helicity-dependent intensity modulation is both spectrally and spatially localized at the nanotube, we interpret the modulation in PL intensity as arising from $\mathrm{CD}$ of the nanotube.

The significant aspect of the CD signal we observe is its magnitude. We define the polarization as $\rho=$ $\left(I_{\mathrm{RCP}}-I_{\mathrm{LCP}}\right) /\left(I_{\mathrm{RCP}}+I_{\mathrm{LCP}}\right)$, and for the data shown in 

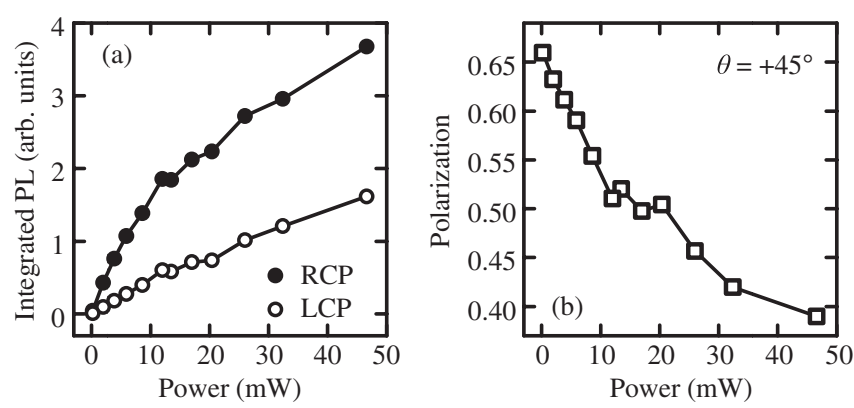

FIG. 2. (a) PL intensity as a function of the laser power for RCP (solid circles) and LCP (open circles), measured at $\theta=+45^{\circ}$ and $\lambda=780 \mathrm{~nm}$ for a CNT with a suspended length of $0.9 \mu \mathrm{m}$. (b) Excitation power dependence of the CD polarization calculated from data in (a).

Fig. 1(b), we obtain $\rho=45 \%$. Furthermore, we find that the apparent polarization increases as the excitation intensity is lowered. On another $(9,7)$ tube, we have investigated the excitation power dependence (Fig. 2). The polarization reaches a value as high as $\rho=65 \%$ at the lowest power that we used. Such a power-dependent behavior is, however, somewhat expected as the PL intensity in CNTs increases sublinearly with excitation power $[17,24,25]$. The PL efficiency is reduced at high excitation rates because of exciton-exciton annihilation, and this is clearly observed for the case of RCP in Fig. 2(a). For LCP, such a sublinear behavior is negligible because the absorption is weaker. The reduction in PL efficiency for RCP results in a smaller difference between PL intensities from what one would expect from the difference in absorption. The apparent reduction of polarization at higher excitation powers is therefore not attributed to changes in the CD of CNTs, and data at low excitation powers are more representative of the actual absorption polarization.

Although it is known that chiral CNTs lack mirror symmetry and can exhibit CD after separation of enantiomers [26,27], the polarization that we observe is about 3 orders of magnitude larger. The fact that the CD signal is vastly larger compared to those observed in ensemble measurements already suggests that the origin of $\mathrm{CD}$ may be different from the intrinsic chirality of CNTs. It turns out that geometrical considerations shed light on the underlying mechanism.

Figures 3(a) and 3(b) show PL spectra taken at two different in-plane incidence angles of $\theta=+40^{\circ}$ and $-40^{\circ}$, respectively. Strikingly, the polarization is inverted for these two geometries. To study such a behavior in more detail, we have performed measurements over a full rotation of the nanotube [Figs. 3(c) and 3(d)]. The integrated PL is maximized at $\theta= \pm 90^{\circ}$ for both RCP and $\mathrm{LCP}$, as expected from strong absorption for linear polarization along the tube axis, but the PL intensities are similar for the two helicities [Fig. 3(e)]. At oblique angles, however, PL intensities are different, and in fact, we find
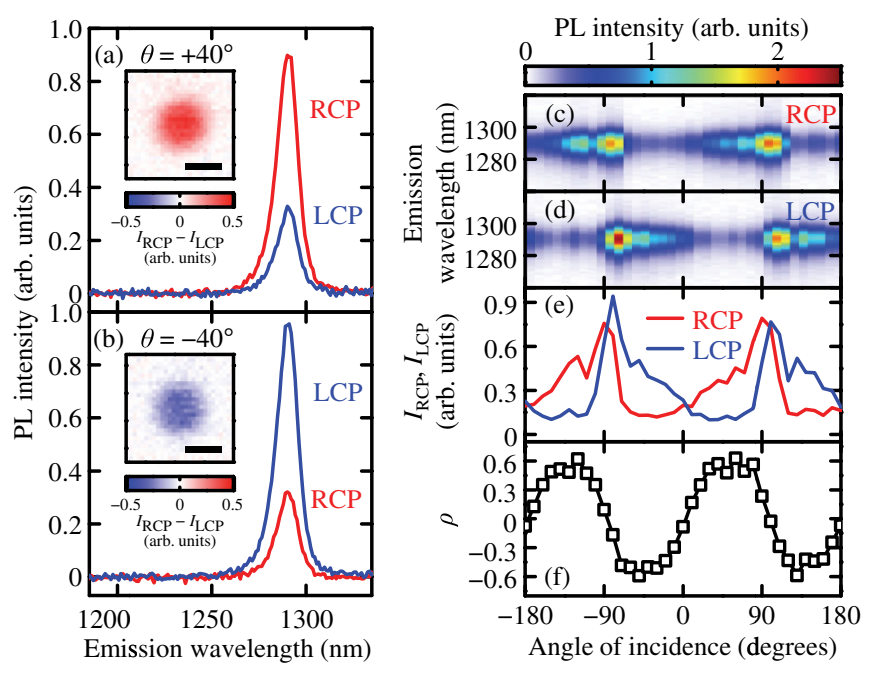

FIG. 3 (color online). (a) and (b) PL spectra taken at $\theta=+40^{\circ}$ and $-40^{\circ}$, respectively, with RCP (red) and LCP (blue). The insets show the difference images. Scale bars are $2 \mu \mathrm{m}$. (c) and (d) In-plane angle dependence of PL spectra for RCP and LCP, respectively. (e) $I_{\mathrm{RCP}}$ (red) and $I_{\mathrm{LCP}}$ (blue) as a function of $\theta$. (f) Polarization calculated from data in (e). Data are taken at $\lambda=780 \mathrm{~nm}$ and $P=2 \mathrm{~mW}$ on a $0.9-\mu \mathrm{m}$-long tube.

that the polarization is strongly dependent on $\theta$ [Fig. 3(f)]. In particular, the signal vanishes when the in-plane optical axis is parallel or perpendicular to the nanotube axis $\left(\theta=0^{\circ}, \pm 90^{\circ}, \pm 180^{\circ}\right)$.

Such geometry-dependent polarization suggests that extrinsic chirality [12] plays an important role, in which the combination of the optical wave vector $\vec{k}$ and the sample breaks the mirror symmetry. If we ignore the presence of the trench, a straight CNT on a substrate has two mirrorsymmetry planes perpendicular to the substrate, where one is normal to the CNT and the other includes the CNT. When the optical axis is outside of these two planes, the mirror symmetry is broken and extrinsic chirality emerges. This symmetry argument is consistent with the angle dependence of the observed polarization.

Statistics also support the interpretation that extrinsic chirality induces the CD. As we expect equal amounts of right- and left-handed CNTs in our samples, the sign of the polarization should be different for half of the tubes if the polarization originates from intrinsic chirality. Figure 4(a) shows the angle-dependent polarization for eight CNTs, where all tubes show positive polarization at $\theta=+45^{\circ}$ and negative polarization at $\theta=-45^{\circ}$. Such a behavior is reproduced in all 25 tubes that we have investigated, including those with different chiral indices.

We note that all tubes in Fig. 4(a) show similar angle dependence, although the angle between these tubes and the trenches ranges from $66^{\circ}$ to $113^{\circ}$. In order to look for any effects of the angle of the trenches, we fit the incidence angle-dependent data to $\rho=A+B \sin \left[2\left(\theta-\theta_{0}\right)\right]$, where $A$ and $B$ are constants and $\theta_{0}$ is the offset angle. In Fig. 4(b), 

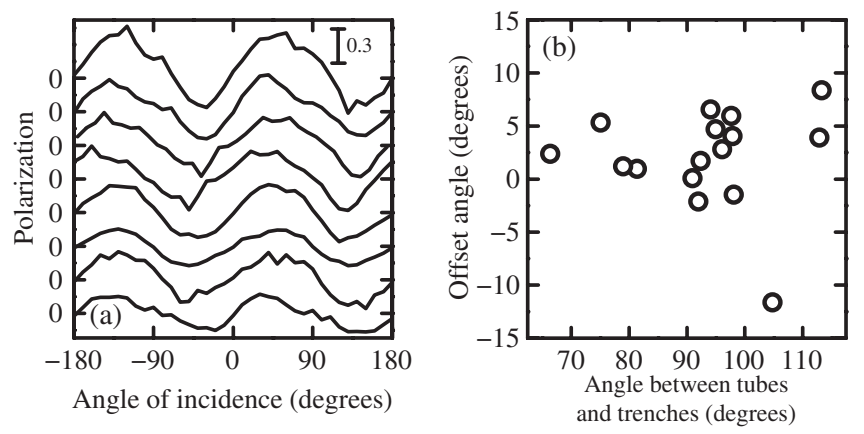

FIG. 4. (a) Angle-dependent polarization for eight representative $(9,7)$ CNTs. The data are offset for clarity. (b) Offset angle plotted as a function of tube angle with respect to the trenches etched along the [110] direction of the Si substrate. Sixteen tubes with a chirality $(9,7)$ are used to generate this plot.

we plot $\theta_{0}$ as a function of the angle between the nanotubes and the trenches, but we do not see a strong correlation. This result shows that the incidence angle with respect to the trench does not play a decisive role in the observed CD.

Now, we propose a microscopic model that explains our observations based on extrinsic chirality. Figure 5(a) shows a schematic of the model. We assume the excitation laser path to be along the $x$ axis, ignore the presence of the trench, and consider the case for $0^{\circ}<\theta<+90^{\circ}$. We do not take into account the intrinsic chirality of the CNT, but we use the fact that linear polarization parallel to the tube

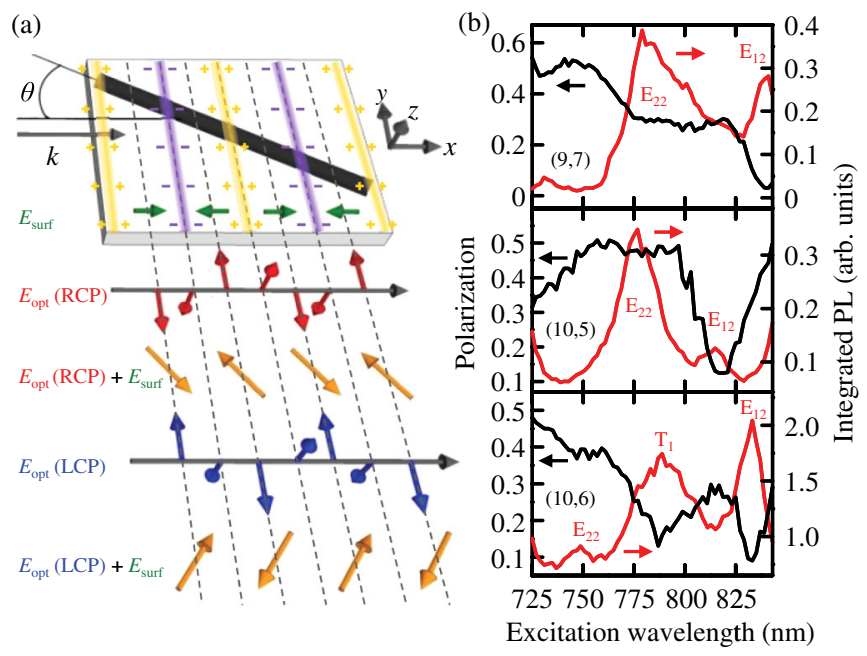

FIG. 5 (color online). (a) A schematic of the model for giant CD as explained in the text. (b) Black curves show CD spectra taken at $\theta=+45^{\circ}$. The excitation powers range from $9 \mathrm{~mW}$ to $28 \mathrm{~mW}$ during wavelength tuning. Red curves show PL excitation spectra taken under a perpendicularly polarized excitation at normal incidence. Top, middle, and bottom panels correspond to a $2.5-\mu$ m-long $(9,7)$ tube, a $3.0-\mu$ m-long $(10,5)$ tube, and a $2.4-\mu \mathrm{m}$-long $(10,6)$ tube, respectively. The excitation spectra are normalized to powers of 170,40 , and $110 \mu \mathrm{W}$ for the $(9,7)$, $(10,5)$, and $(10,6)$ tubes, respectively. The peaks are assigned according to Ref. [28]. axis dominates in the absorption process [17,22,23]. In Fig. 5(a), a snapshot of the spatially varying optical electric field $\vec{E}_{\text {opt }}$ is shown for both helicities. At positions where $\vec{E}_{\text {opt }}$ is normal to the substrate, there will be an induced charge on the surface that generates in-plane surface electric fields $\vec{E}_{\text {surf }}$ parallel to $x$. We note that $\vec{E}_{\text {surf }}$ does not depend on the helicity of the excitation.

At positions where $\vec{E}_{\text {opt }}$ is parallel to the substrate, the sum $\vec{E}_{\text {opt }}+\vec{E}_{\text {surf }}$ can have considerably different orientations for the two helicities. For RCP, the total fields will tend to be oriented at a smaller angle to the nanotube axis, while for LCP, they tend to be oriented more perpendicularly to the nanotube axis. Essentially, the presence of the substrate converts the circular polarization to in-plane linear polarization, enhancing or reducing the absorption depending on the helicity. Such a conversion is distinctly different from conventional means, as the resultant polarization corresponds to a different direction of the optical wave vector.

This model can qualitatively explain the angle dependence of the CD. Inverting the angle will swap the roles of $\mathrm{RCP}$ and LCP, and the polarization changes sign. At $\theta=0^{\circ}$ and $\pm 90^{\circ}$, the total field for RCP and LCP becomes symmetric with respect to the nanotube axis, and therefore the $\mathrm{CD}$ vanishes. In addition, $\mathrm{CD}$ spectra are also consistent with the proposed model. As linearly polarized absorption parallel to the tubes is required in this model, the effect is expected to weaken at the $E_{12}$ transition where the absorption polarization is reduced [28,29]. Indeed, we observe broadband polarization with some decrease near $E_{12}$ transitions [Fig. 5(b)]. Such a wavelength dependence is in contrast to ensemble measurements that have shown CD peaks at $E_{22}$ and $E_{33}$ transitions [26,27]. We also observe that the $\mathrm{CD}$ does not change sign throughout the spectra, indicating that this mechanism is different from exciton-coupled CD in chiral molecules [2].

Furthermore, the magnitude of the observed CD can be quantitatively explained by this model. The field inside the substrate is given by $\left(\epsilon_{0} / \epsilon_{\text {sub }}\right) E_{\text {opt }}$, where $\epsilon_{0}$ is the vacuum permittivity and $\epsilon_{\text {sub }}$ is the substrate permittivity, so the surface charge density is

$$
\sigma(x)=\epsilon_{0} E_{\mathrm{opt}}\left(1-\frac{\epsilon_{0}}{\epsilon_{\mathrm{sub}}}\right) \sin (k x) .
$$

Then, the surface field at $x=0$ is given by

$$
E_{\mathrm{surf}}(x=0)=\int \frac{\sigma(x)}{2 \pi \epsilon_{0} x} d x=\frac{E_{\mathrm{opt}}}{2}\left(1-\frac{\epsilon_{0}}{\epsilon_{\mathrm{sub}}}\right),
$$

and using a value of $\epsilon_{\text {sub }}=12 \epsilon_{0}$ for $\mathrm{Si}$, we obtain $E_{\text {surf }}=0.46 E_{\text {opt }}$. The angles between the total fields and the nanotube axis for RCP and LCP become $20^{\circ}$ and $110^{\circ}$, respectively, and this results in a polarization of $76 \%$ at $\theta=45^{\circ}$, assuming perfect linearly polarized absorption by 


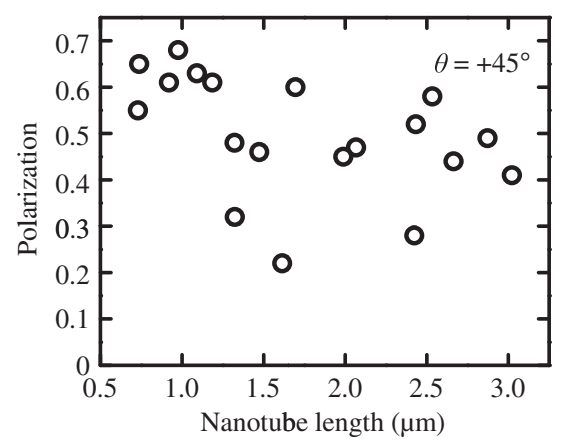

FIG. 6. Dependence of CD polarization on the suspended length of $(9,7)$ CNTs. Data are taken with $\theta=+45^{\circ}$, $\lambda=780 \mathrm{~nm}$, and $P=5 \mathrm{~mW}$.

the nanotube. This is in good agreement with the experimentally observed polarization of $65 \%$, considering reduced absorption polarization in real nanotubes.

We have also investigated suspended length dependence of the polarization. As the presence of the substrate is the essential origin of $\mathrm{CD}$ in our model, the polarization is expected to decrease as the suspended length of the CNT increases. The suspended part does not have the substrate underneath, and locally it possesses mirror symmetry so it should contribute less to CD. Such a dependence is, more or less, observed in Fig. 6, but against our expectation, the polarization remains for lengths much longer than a wavelength. Further investigation is necessary to clarify this weak dependence.

Our results have important implications for the detection of $\mathrm{CD}$ in other nanoscale systems, including single molecules. The polarization conversion by extrinsic chirality causes an object with linear dichroism to appear as if it has circular dichroism, and this has not been considered in previous attempts to detect CD in individual molecules [5-9]. As the extrinsic chirality effects can completely overwhelm the response from intrinsic chirality, it is important to identify the geometry and the strength of linear dichroism for the objects under investigation. In particular, incidence-angle dependence measurements should be able to separate the extrinsic and intrinsic chirality contributions.

It is remarkable that extrinsic chirality can induce such a giant response in a system as simple as a nanotube suspended over a trench, and it may find applications in optical devices for polarization control. Although mode conversion would be necessary, an array of nanotubes oriented at $45^{\circ}$ on a waveguide would preferentially absorb one helicity and act as a filter for circularly polarized light. The same device can be used to convert polarization because linearly polarized light at the input would end up circularly polarized at the output by an extinction of the other helicity. Such a device should still function if nanotubes are replaced by semiconductor nanowires, as they also have strong linear dichroism. The proposed mechanism for the giant $\mathrm{CD}$ relies on the surface fields, and it may also be possible to use them for other types of polarization conversion. We hope that our findings will lead to new techniques for polarization manipulation at the nanoscale.

We thank K. Konishi and H. Tamaru for helpful discussions and acknowledge support from KAKENHI (Grants No. 21684016, No. 23104704, No. 24340066, No. 24654084), SCOPE, Asahi Glass Foundation, KDDI Foundation, and the Photon Frontier Network Program of MEXT, Japan. The samples were fabricated at the Center for Nano Lithography \& Analysis at The University of Tokyo.

[1] E. Hecht, Optics (Addison-Wesley, San Francisco, 2002), 4th ed.

[2] Circular Dichroism: Principles and Applications, edited by N. Berova, K. Nakanishi, and R. W. Woody (Wiley-VCH, New York, 2000), 2nd ed.

[3] E. Hendry, T. Carpy, J. Johnston, M. Popland, R. V. Mikhaylovskiy, A. J. Lapthorn, S. M. Kelly, L. D. Barron, N. Gadegaard, and M. Kadodwala, Ultrasensitive Detection and Characterization of Biomolecules using Superchiral Fields, Nat. Nanotechnol. 5, 783 (2010).

[4] Y. Tang and A. E. Cohen, Enhanced Enantioselectivity in Excitation of Chiral Molecules by Superchiral Light, Science 332, 333 (2011).

[5] R. Hassey, E. J. Swain, N. I. Hammer, D. Venkataraman, and M. D. Barnes, Probing the Chiroptical Response of a Single Molecule, Science 314, 1437 (2006).

[6] Y. Tang, T. A. Cook, and A. E. Cohen, Limits on Fluorescence Detected Circular Dichroism of Single Helicene Molecules, J. Phys. Chem. A 113, 6213 (2009).

[7] M. D. Barnes, R. H. Paradise, E. Swain, D. Venkataraman, and N. I. Hammer, Comment on Limits on Fluorescence Detected Circular Dichroism of Single Helicene Molecules, J. Phys. Chem. A 113, 9757 (2009).

[8] A. Cohen and Y. Tang, Reply to Comment on 'Limits on Fluorescence Detected Circular Dichroism of Single Helicene Molecules', J. Phys. Chem. A 113, 9759 (2009).

[9] A. Cyphersmith, S. Surampudi, M. J. Casey, K. Jankowski, D. Venkataraman, and M. D. Barnes, Chiroptical Dissymmetries in Fluorescence Excitation from Single Molecules of (M-2) Helicene Dimers, J. Phys. Chem. A 116, 5349 (2012).

[10] M. Kuwata-Gonokami, N. Saito, Y. Ino, M. Kauranen, K. Jefimovs, T. Vallius, J. Turunen, and Y. Svirko, Giant Optical Activity in Quasi-Two-Dimensional Planar Nanostructures, Phys. Rev. Lett. 95, 227401 (2005).

[11] J. K. Gansel, M. Thiel, M. S. Rill, M. Decker, K. Bade, V. Saile, G. von Freymann, S. Linden, and M. Wegener, Gold Helix Photonic Metamaterial as Broadband Circular Polarizer, Science 325, 1513 (2009).

[12] E. Plum, X.-X. Liu, V. A. Fedotov, Y. Chen, D. P. Tsai, and N. I. Zheludev, Metamaterials: Optical Activity without Chirality, Phys. Rev. Lett. 102, 113902 (2009). 
[13] C. M. Soukoulis and M. Wegener, Past Achievements and Future Challenges in the Development of ThreeDimensional Photonic Metamaterials, Nat. Photonics 5, 523 (2011).

[14] J. B. Pendry, A Chiral Route to Negative Refraction, Science 306, 1353 (2004).

[15] S. Maruyama, R. Kojima, Y. Miyauchi, S. Chiashi, and M. Kohno, Low-Temperature Synthesis of High-Purity Single-Walled Carbon Nanotubes from Alcohol, Chem. Phys. Lett. 360, 229 (2002).

[16] S. Imamura, R. Watahiki, R. Miura, T. Shimada, and Y. K. Kato, Optical Control of Individual Carbon Nanotube Light Emitters by Spectral Double Resonance in Silicon Microdisk Resonators, Appl. Phys. Lett. 102, 161102 (2013).

[17] S. Moritsubo, T. Murai, T. Shimada, Y. Murakami, S. Chiashi, S. Maruyama, and Y. K. Kato, Exciton Diffusion in Air-Suspended Single-Walled Carbon Nanotubes, Phys. Rev. Lett. 104, 247402 (2010).

[18] S. Yasukochi, T. Murai, S. Moritsubo, T. Shimada, S. Chiashi, S. Maruyama, and Y. K. Kato, Gate-Induced Blueshift and Quenching of Photoluminescence in Suspended Single-Walled Carbon Nanotubes, Phys. Rev. B 84, 121409(R) (2011).

[19] R. Watahiki, T. Shimada, P. Zhao, S. Chiashi, S. Iwamoto, Y. Arakawa, S. Maruyama, and Y. K. Kato, Enhancement of Carbon Nanotube Photoluminescence by Photonic Crystal Nanocavities, Appl. Phys. Lett. 101, 141124 (2012).

[20] S. M. Bachilo, M. S. Strano, C. Kittrell, R. H. Hauge, R. E. Smalley, and R. B. Weisman, Structure-Assigned Optical Spectra of Single-Walled Carbon Nanotubes, Science 298, 2361 (2002).
[21] Y. Ohno, S. Iwasaki, Y. Murakami, S. Kishimoto, S. Maruyama, and T. Mizutani, Chirality-Dependent Environmental Effects in Photoluminescence of Single-Walled Carbon Nanotubes, Phys. Rev. B 73, 235427 (2006).

[22] A. Hartschuh, H. N. Pedrosa, L. Novotny, and T. D. Krauss, Simultaneous Fluorescence and Raman Scattering from Single Carbon Nanotubes, Science 301, 1354 (2003).

[23] J. Lefebvre, J. M. Fraser, P. Finnie, and Y. Homma, Photoluminescence from an Individual Single-Walled Carbon Nanotube, Phys. Rev. B 69, 075403 (2004).

[24] F. Wang, G. Dukovic, E. Knoesel, L. E. Brus, and T. F. Heinz, Observation of Rapid Auger Recombination in Optically Excited Semiconducting Carbon Nanotubes, Phys. Rev. B 70, 241403 (2004).

[25] Y.-F. Xiao, T. Q. Nhan, M. W. B. Wilson, and J. M. Fraser, Saturation of the Photoluminescence at Few-Exciton Levels in a Single-Walled Carbon Nanotube under Ultrafast Excitation, Phys. Rev. Lett. 104, 017401 (2010).

[26] X. Peng, N. Komatsu, S. Bhattacharya, T. Shimawaki, S. Aonuma, T. Kimura, and A. Osuka, Optically Active Single-Walled Carbon Nanotubes, Nat. Nanotechnol. 2, 361 (2007).

[27] S. Ghosh, S. M. Bachilo, and R. B. Weisman, Advanced Sorting of Single-Walled Carbon Nanotubes by Nonlinear Density-Gradient Ultracentrifugation, Nat. Nanotechnol. 5, 443 (2010).

[28] J. Lefebvre and P. Finnie, Polarized Photoluminescence Excitation Spectroscopy of Single-Walled Carbon Nanotubes, Phys. Rev. Lett. 98, 167406 (2007).

[29] Y. Miyauchi, H. Ajiki, and S. Maruyama, Electron-Hole Asymmetry in Single-Walled Carbon Nanotubes Probed by Direct Observation of Transverse Quasidark Excitons, Phys. Rev. B 81, 121415(R) (2010). 